\title{
LEC-AODV Routing Protocol Based on Load and Energy Classification
}

\author{
Dengyin ZHANG ${ }^{1, a}$ Zhaoguo $\mathrm{CHEN}^{2, b}$ \\ ${ }^{1,2}$ Key Lab of Broadband Wireless Communication and Sensor Network Technology \\ (Nanjing University of Posts and Telecommunications), Ministry of Education, \\ Nanjing 210003, China \\ azhangdy@njupt.edu.cn, ${ }^{\mathrm{b}} \mathrm{czglenovo@163.com}$
}

Key Words : AODV, classification, load, energy, QoS, tactical MANETs

\begin{abstract}
Now widely used AODV routing protocol does not take into account the node's load size and energy level. During the route discovery process, AODV routing protocol often has the blindness and makes it easy to bring about network congestion and consume some node's energy excessively. To solve this problem, this paper presents a new LEC-AODV routing protocol based on load and energy classification, which makes a different response according to the node's load size and energy level. Simulation results show that the protocol does not significantly increase the complexity of traditional AODV routing algorithm when packet delivery ratio, average end-to-end delay and network lifetime and other indicators have improved in varying degrees. At the same time it can bypass the nodes with heavy load and low energy, thereby achieve a certain degree of flow and energy balance and successfully solve the QoS problem in tactical MANETs.
\end{abstract}

\section{Introduction}

Tactical MANETs which are composed of soldiers, ground vehicles, sensors and command centers are self-organized, highly dynamic, multi-hop wireless mobile ad hoc networks, and will play an significant role in future information warfare. Tactical MANETs are characterized by large traffic flow and high dynamic changes which make it prone to congestion and nodes died early [1]. Therefore, the node's load and energy play an important part in tactical MANETs. If a node has heavy load and low energy, it will die early. Even worse, its death will affect the QoS across the networks when the node is a core node. Based on load and energy classification, this paper presents a new LEC-AODV routing protocol to find a stable and reliable route, which effectively bypass the nodes with heavy load and low energy. According to the node's different state this routing protocol makes different route response to meet the QoS requirements of tactical MANETs.

\section{AODV Routing Protocol}

AODV routing protocol is one of the routing protocol recommended by IETF MANETs working group [2]. It is an on-demand routing protocol, with a simple and superior performance characteristics, but the active routing protocol requires periodic broadcast with heavy routing load. Therefore, AODV routing protocol has been widely used in MANETs. During the route found period, AODV routing protocol often has blindness and do not considers the node's load size and energy level, so the congestion occurs frequently and nodes died early, which seriously affects the communication quality of tactics MANETs. As AODV routing protocol has many disadvantages, the existing technology improvement program are the following aspects: Based on bandwidth estimation, Lei Chen [3] proposed a QoS-Aware routing protocol that incorporated an admission control scheme and a feedback scheme to meet the QoS requirements of real-time applications. 
Yaser K [4] proposed a Enhanced VON-AODV based on delayed rebroadcast Scheme. By monitoring the node's queue length it will forwards PREQ message with linear delay, so that the nodes with low load maybe response the routing more positively. Arjan D [5,6] proposed an Energy-Aware routing protocol and achieved a good performance in terms of network lifetime by balancing the energy among all the nodes.

Most of above researches only solve a partial QoS problem of AODV at a particular level. They do not consider the whole node's state to format a suitable QoS solution for tactical MANETs. With the characteristics of tactical MANETs this paper unifies the node's load size and energy level to propose a new routing protocol that effectively solves the QoS problem for tactical MANETs.

\section{LEC-AODV Routing Protocol}

In view that the existing improved AODV routing protocols have many shortcoming, this improved LEC-AODV protocol divides the node's load and energy into different level which as the basis for route discovery process. The node's different level will decide that a PREQ message is forwarded immediately, forwarded delay or discard by a certain probability, so that it can bypass the heavy load and low energy nodes that will balance the load and energy among all the nodes.

\section{A. Load and Energy Information Collection}

There are two methods to collect node's load information: One is bandwidth utilization which can be estimated through periodic monitoring the channel busy time of data link layer [7]. If the current time is defined as $t$, a cycle is defined as $T$, the channel busy time is defined as which includes the following three states: transmitting or receiving a packet, receiving an RTS or CTS message from another node and sensing a busy carrier with signal strength larger than a certain threshold. Taking into account the impact of unexpected data flow, we can use the method of weighted moving average to predict the current bandwidth utilization:

$$
L B U(t)=\alpha \times L B U(t-T)+((1-\alpha) \times L B U(\text { busy })) / T
$$

Where $L B U(t-T)$ is the previous bandwidth utilization and weight factor $\alpha \in(0,1)$. The simulation analysis showed that when $\alpha=0.2$, Eq. 1 predicted the bandwidth utilization that is closest to the actual bandwidth utilization. The other is queue utilization [8]. Suppose the current monitored queue length is defined as $q(t)$, the maximum queue length is defined as $Q$. We also use the method of weighted moving average to predict the current queue utilization:

$$
Q U(t)=\beta \times Q U(t-T)+((1-\beta) \times q(t)) / Q
$$

Where $Q U(t-T)$ is the previous queue utilization and weight factor $\beta \in(0,1)$. The simulation analysis showed that $\beta=0.2$, Eq. 2 predicted the queue utilization that is closest to the actual queue utilization. Based on the above two methods, the node's load can be made:

$N C(t)=\gamma \times L B U(t)+(1-\gamma) \times Q U(t)$

Where $\gamma$ is the weight factor $\gamma \in(0,1)$. Simulation results show that, when a node appears a packet waited to be forwarded, the possibility of congestion would increase greatly. In order to better reflect the weight of queue utilization in the node's load, the weight factor $\gamma=0.4$ and achieves a good performance.

The residual energy information of the current node can be gathered by direct access the routing layer. Assuming that the residual energy of the current node is $E(t)$, the initial energy of node is $E$ (Origin) . So the ratio of residual energy is:

$$
E U(t)=E(t) / E(\text { Origin })
$$

B. LEC-AODV Routing Algorithm

During the route discovery process, the intermediate nodes will decide whether a PREQ message is forwarded immediately, forwarded delay or discard by a certain probability based on the collected information of load and energy. To make the current node accurately response the PREQ message 
according to its load and energy, the load and energy of node is divided into three levels: when $N C(t)>0.8$ is defined as the congestion, $0.4<N C(t)<0.8$ is defined as heavy load, $N C(t)<0.4$ is defined as light load; When $E U(t)>0.5$ is defined as energy level 3, $0.1<E U(t)<0.5$ is defined as energy level 2, $E U(t)<0.1$ is defined as lowest energy level 1 .

When the load and energy of nodes is divided into three levels, the intermediate nodes will response by the classification of the various situations that may arise and solve the problem of congestion and nodes died early. The following steps are LEC-AODV route discovery process algorithm:

(1) When the intermediate node receives a PREQ message, first check whether it has received the same PREQ message recently. If yes, it will discard it. Otherwise, it will query and calculation its load and energy information using Eq.1, 2, 3 and 4. Then it will determine its load and energy levels by the collected load and energy information.

(2) If the intermediate node is in a state of congestion, it will discard the PREQ message directly.

(3) If the intermediate node is in a heavy load state, there are following three cases to deal with. When the energy level is 3, the intermediate node forwards the PREQ message immediately. When the energy level is 2, the intermediate node will forward the PREQ message after a short time. When the energy level is 1, the intermediate node discards the PREQ message by a certain probability P. If it fails to drop the PREQ message, it will delay a short time to forward the PREQ message.

(4) If the intermediate node is in a light load state, there are following three cases to deal with. When the energy level is greater than 1, the intermediate node forwards the PREQ message immediately. When the energy level is 1 , the intermediate node discards the PREQ message by a certain probability Q. If it fails to drop the PREQ message, the PREQ message will be forwarded after a short time. The selection of the probability $\mathrm{P}$ and $\mathrm{Q}$ relates by the specific network. In this paper, the networks take the best performance when $\mathrm{P}=0.8$ and $\mathrm{Q}=0.6$.

The purpose of using delay function during the route discovery process is to bypass the nodes with heavy load and low energy, so we can reduce the congestion and improve network lifetime. Due to dynamic nature of traffics and movements in MANETs, the performance of LEC-AODV will be greatly influenced by which delay function is being used. We experimented the following three kinds of delay functions: liner, convex and concave functions [9]. Experiments showed that they delay to forward PREQ only by the residual energy and do not consider the load, so it will not avoid the occurrence of congestion when the node have low energy and heavy load. However, the above delay functions do not achieve good performance when the node has high energy or low energy. Considering that the paper selects the node's load and energy to measure the availability of the current node, we use the following delay function:

$$
\operatorname{delay}(t)=\delta \times(1-n a(t))^{3} /\left(1+\varepsilon \times(1-n a(t))^{3}\right)
$$

Where $\varepsilon$ denotes curvature coefficient, $\delta$ denotes delay coefficient, $n a(t)$ denotes the node's available utilization:

$$
n a(t)=0.5 \times(1-N C(t))+0.5 \times E U(t)
$$

Simulation results show that the networks take the best performance and rarely occur the phenomenon of congestion and nodes died early when $\varepsilon=10$ and $\delta=0.5$. (To maximize the network performance the selection of these parameters are determined by the specific circumstances of the networks.) 
The above improved scheme is mainly found in the route discovery process, and improved LEC-AODV routing algorithm only increases the sentences of inquiries and multiple choices in the original algorithm. The complexity of improved algorithm is as the same level as the original algorithm, so the LEC-AODV routing algorithm does not significantly increase the complexity when compared with the traditional AODV routing algorithm.

\section{Simulation and Analysis}

A. Simulation Environment

We have implemented the LEC-AODV routing protocol and compared with the original AODV routing protocol in the ns-2 simulator [10]. The specific parameters of simulation environment are configured as follows: A total of 50 nodes were simulated for 120 s over a network space of $1000 \times 1000 \mathrm{~m}$. The network traffic was modeled as $20 \mathrm{CBR}$ sources with data sent in 512 byte packets, and it sends packets at a requested rate between 6 and 12 packet/s. Movement patterns were generated based on a random waypoint model and each value of pause time is $5 \mathrm{~s}$. The initial energy of all the nodes is 25J. The IEEE 802.11 Distributed Coordination Function (DCF) is used as the medium access protocol. The interface queue is a 50-packet drop-tail priority queue.

B. The Performance of Analysis

We evaluated the following four performance metrics to compare the performance of AODV to LEC-AODV: packet delivery ratio, average end-end delay, routing cost and network lifetime.

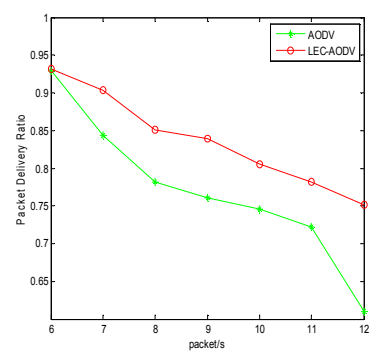

Fig.1 Packet Delivery Ratio

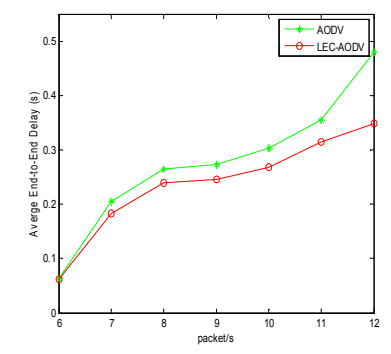

Fig.2 Average End-to-End Delay

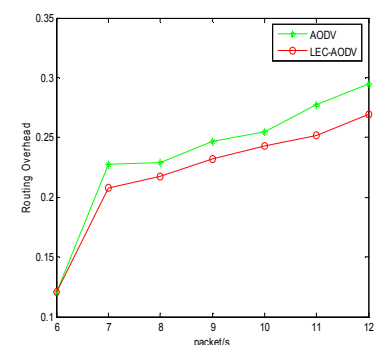

Fig.3 Routing

Overhead

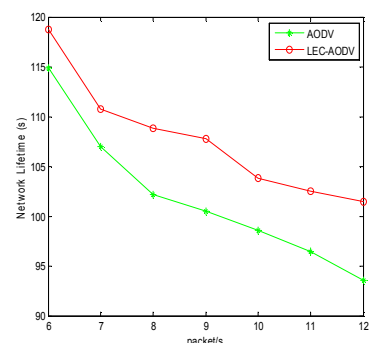

Fig.4 Network Lifetime

\section{(1)Packet Delivery Ratio}

Fig. 1 shows the contrast curves of packet delivery ratio at different speeds between LEC-AODV and AODV. As shown in Fig.1, in the case of light network load, due to network congestion does not occur at this time, so the performance between AODV and LEC-AODV has little difference. The packet delivery ratio of traditional AODV declines soon when the load of networks increases. However, the packet delivery ratio of LEC-AODV does not significantly fell. When the rate of sending packet exceeds 12 packet / s, the packet delivery ratio is still able to preserve more than $75 \%$.

(2) Average End-to-End Delay

Average end-to-end delay is defined as sum of the delays of each CBR packet received divided by number of CBR packets received. Fig. 2 indicates the contrast curves of average end-to-end delay at different speeds between LEC-AODV and AODV. When the networks have light load, the gap of average end-to-end delay is not large between AODV and LEC-AODV. But when the networks have heavy load, the average end-to-end delay of traditional AODV increases dramatically, and the improved routing protocol does not. This is because LEC-AODV takes into account the current load of network and can bypass the nodes with heavy load, so it make the average end-to-end delay increase less than traditional AODV. 


\section{(3) Routing Overhead}

Routing overhead is defined as sum of the route packets divided by sum of the CBR packets. Fig. 3 depicts the contrast curves of routing overhead at different speeds between LEC-AODV and AODV. As expected in Fig.3, The improved LEC-AODV does better in routing overhead than traditional AODV. Because of the many nodes died early makes the entire networks topology change which will lead to a large number of routing overhead packets. However, considering the energy of current node the improved LEC-AODV makes the low energy of node involve in the route discovery process as little as possible, thus greatly reducing the routing overhead.

(4) Network Lifetime

Network lifetime is defined as the time when a node runs out of its own energy for the first time. Fig.4 shows the contrast curves of network lifetime at different speeds between LEC-AODV and AODV. Whether the networks have heavy load or light load, the improved AODV gets better than before in the network lifetime. This is mainly because the improved LEC-AODV can avoid the nodes with heavy load and low energy according to the load and energy classification, thus greatly extending the lifetime of the networks and improving the QoS of tactical MANETs.

\section{Summary}

In this paper, we propose a LEC-AODV routing protocol based on load and energy classification for tactical MANETs. The concept of the proposed approach is to find a stable and reliable route through dividing the load and energy into different levels which will bypass the nodes with heavy load and low energy during the route discovery. Simulation results show LEC-AODV has balanced load and energy effectively and achieves far better performance than AODV in tactical MANETs.

\section{References}

[1] Fossa C, Macdonald T, Internetworking Tactical MANETs, the 2010 Military Communications Conference (2010) 1034-1039

[2] Information on http://www.ieft.org/rfc/rfc3561.txt

[3] Lei Chen, Wendi B, QoS-Aware Routing Based on Bandwidth Estimation for Mobile Ad Hoc Networks, IEEE Journal on Selected Areas in Communications 23(4) (2005) 561-572

[4] Yaser K, Ghadeer O, Asmahan A, Enhanced VON-AODV Based on Delayed Rebroadcast Scheme, Proceedings of the 4th ACM workshop on Performance monitoring and measurement of heterogeneous wireless and wired networks (2009) 78-83

[5] Jin-Man K, Jong-Wook J, AODV based Energy Efficient Routing Protocol for Maximum Lifetime in MANET, Proceedings of AICT/ICIW (2006) 77-82

[6] Ming Liu, Jiannong Cao, Guihai Chen, An Energy-Aware Routing Protocol in Wireless Sensor Networks, Senors (2009)445-462

[7] Ningning $H$, Peter S, Evaluation and Characterization of Available Bandwidth Probing Techniques, IEEE JOURNAL ON SELECTED AREAS IN COMMUNICATIONS 21(8) (2003) 879-894

[8] Yang S, Thomas G, WXCP: Explicit Congestion Control for Wireless Multi-hop Networks, Lecture Notes in Computer ScienceHH 3552 (2005) 263-278

[9] Li Layuan, Li Chunlin, Yuan Peiyan, An Energy Level Based Routing Protocol in Ad Hoc Networks, Proceedings of the IEEE/WIC/ACM International Conference on Intelligent Agent Technology (2006) 306-312

[10] Information on http://www.isi.edu/nsnam/ns/doc/index.html 